\title{
Deep transcriptome sequencing provides new insights into the structural and functional organization of the wheat genome
}

\author{
Lise Pingault ${ }^{1,2}$, Frédéric Choulet ${ }^{1,2}$, Adriana Alberti ${ }^{3}$, Natasha Glover ${ }^{1,2,6}$, Patrick Wincker ${ }^{3,4,5}$,
} Catherine Feuillet ${ }^{1,2,7}$ and Etienne Paux ${ }^{1,2^{*}}$

\begin{abstract}
Background: Because of its size, allohexaploid nature, and high repeat content, the bread wheat genome is a good model to study the impact of the genome structure on gene organization, function, and regulation. However, because of the lack of a reference genome sequence, such studies have long been hampered and our knowledge of the wheat gene space is still limited. The access to the reference sequence of the wheat chromosome 3B provided us with an opportunity to study the wheat transcriptome and its relationships to genome and gene structure at a level that has never been reached before.

Results: By combining this sequence with RNA-seq data, we construct a fine transcriptome map of the chromosome 3B. More than 8,800 transcription sites are identified, that are distributed throughout the entire chromosome. Expression level, expression breadth, alternative splicing as well as several structural features of genes, including transcript length, number of exons, and cumulative intron length are investigated. Our analysis reveals a non-monotonic relationship between gene expression and structure and leads to the hypothesis that gene structure is determined by its function, whereas gene expression is subject to energetic cost. Moreover, we observe a recombination-based partitioning at the gene structure and function level.

Conclusions: Our analysis provides new insights into the relationships between gene and genome structure and function. It reveals mechanisms conserved with other plant species as well as superimposed evolutionary forces that shaped the wheat gene space, likely participating in wheat adaptation.
\end{abstract}

\section{Background}

In angiosperms, genome size is extremely variable, ranging from $63 \mathrm{Mb}$ in Genlisea margaretae to 148,900 Mb in Paris japonica, that is, a 2,400-fold difference [1]. By contrast, the gene content seems relatively constant, with an average number of 30,000 and a two- to threefold difference per diploid genome [2,3]. As a consequence, the gene space organization differs strikingly from one genome to another. For example, plants with small genomes such as Arabidopsis thaliana (125 Mb) and Brachypodium distachyon (272 Mb) exhibit an even

\footnotetext{
* Correspondence: etienne.paux@clermont.inra.fr

'INRA UMR1095 Genetics, Diversity and Ecophysiology of Cereals, 5 chemin de Beaulieu, 63039 Clermont-Ferrand, France

${ }^{2}$ University Blaise Pascal UMR1095 Genetics, Diversity and Ecophysiology of Cereals, 5 chemin de Beaulieu, 63039 Clermont-Ferrand, France

Full list of author information is available at the end of the article
}

distribution of their genes along their chromosomes [4] whereas for plants with intermediate size genomes such as Populus trichocarpa (485 Mb) and Vitis vinifera (487 Mb), alternation between high gene density regions and low gene density regions is observed [5,6]. This tendency is even stronger in plants with large genomes such as Glycine $\max (1,115 \mathrm{Mb})$ and Zea mays $(2,300 \mathrm{Mb})$ in which a positive gradient of gene density from the centromere to the telomeres has been observed $[7,8]$. Beside the overall organization of genes, several studies revealed a non-random distribution of genes along chromosomes, resulting in clusters of genes sharing the same expression profile, the same function or involved in the same metabolic pathway [9-15]. In addition, relationships between gene structure and expression were reported in various organisms [16-18]. Altogether, these studies suggest a high degree of organization in gene 
space and interplay between genome and gene structure, function, and regulation.

With 220 million hectares, bread wheat (Triticum aestivum L.) is the most widely grown and consumed crop worldwide providing staple food for $30 \%$ of the world population. Beside its socioeconomic importance, bread wheat is also a good model for studying complex genome species. Indeed, with its large 17-Gb, allohexaploid $(6 \mathrm{x}=2 n=42$, AABBDD) and highly repetitive ( $>80 \%$ transposable elements) genome, wheat is one of the most complex crop species. Other species share some of these features, but none of them, at least among cultivated species, combine the three. For example, the loblolly pine genome is the largest genome sequenced so far (22 Gb) but it is diploid [19]. Cotton is a polyploid species but has a smaller genome $(2.5 \mathrm{~Gb})[20]$ and so far only wild diploid relatives were sequenced [21,22]. The maize and sorghum genomes are highly repetitive but are diploid and smaller in size $[7,8]$.

The wheat gene space organization and expression have been extensively investigated in the past decades. Many expression analyses have been conducted using either microarrays or RNA-seq but most of them were aiming at deciphering specific processes, such as grain development or response to stresses (for examples, see [23-27]). Other studies aimed at studying the gene space organization and reported on the existence of a gene gradient along the centromere-telomere axis as well as an organization of genes in small gene islands and co-expression/co-function clusters (for examples, see [28-30] and references therein). However, very few of these studies really investigated the relationships between genome and gene structure and function, mainly because of the lack of a reference genome sequence. The access to physical maps of wheat chromosomes provided the first opportunities to study gene regulation with respect to their physical position [29] although there were still limited to efficiently address this question. Recently, several initiatives aimed at generating draft genome sequences of hexaploid wheat or its diploid progenitors $[21,22,31,32]$. While they provided a quite comprehensive catalogue of wheat genes as well as novel data on gene evolution and expression, the highly fragmented nature of the sequence assemblies limits our ability to decipher the relationships between genome organization and gene regulation.

Recently, we have produced a 774-Mb reference sequence of the hexaploid wheat chromosome 3B [33]. Sequence annotation predicted 7,264 genes that were distributed along the chromosome with a gradient of density from centromere to telomeres. The distribution of structural and functional features along the chromosome revealed partitioning correlated with meiotic recombination. Three main regions were identified: two distal regions of $68 \mathrm{Mb}$ (region $\mathrm{R} 1$; from 1 to $68 \mathrm{Mb}$ ) and $59 \mathrm{Mb}$ (region $\mathrm{R} 3$; from 715 to $774 \mathrm{Mb}$ ) on the short and long arms, respectively, and a large proximal region of $648 \mathrm{Mb}$ (region $\mathrm{R} 2$; from 68 to $715 \mathrm{Mb}$ ) spanning the centromere. In addition, we delineated a $122-\mathrm{Mb}$ central region (from 265 to $387 \mathrm{Mb}$ ), enriched in centromere-specific transposable elements, as the centromericpericentromeric region of chromosome 3B.

Here, we report a detailed analysis of the chromosome 3B transcriptional landscape. By combining deep transcriptome sequencing data covering the whole plant development with the reference sequence of the chromosome, we identified transcriptionally active regions distributed throughout the entire chromosome. Relationships between genome and gene structure and function revealed different mechanisms governing the gene space organization, regulation, and evolution.

\section{Results and discussion}

\section{Chromosome 3B contains more than 8,800 transcriptionally active regions}

To study the expression profiles of hexaploid wheat chromosome 3B genes during the life cycle of a wheat plant and establish a transcriptome atlas for this chromosome, deep transcriptome sequencing was conducted in duplicates in 15 wheat samples corresponding to five different organs (leaf, shoot, root, spike, and grain) at three developmental stages each [28]. Strandnon-specific and strand-specific libraries were used to produce 2.52 billion paired-end reads $(232 \mathrm{~Gb})$ and 615.3 single-end reads $(62 \mathrm{~Gb})$, respectively. The reads were then mapped to the chromosome $3 \mathrm{~B}$ reference sequence [33], without allowing for any mismatches in order to discriminate chromosome $3 \mathrm{~B}$ expressed genes from homoeologous and paralogous copies. Eventually, $3.66 \%$ of reads mapped onto chromosome $3 \mathrm{~B}$ of which $98 \%$ were mapped uniquely. Ninety-five percent of the reads matched sequences annotated as genic regions whereas the remaining 5\% mapped to regions where no protein-coding gene was predicted by the annotation [33].

Within the 774.4 $\mathrm{Mb}$ comprising the pseudomolecule of chromosome 3B, 8,877 transcriptionally active regions (TARs) were identified, corresponding to an average density of one TAR every $87 \mathrm{~kb}$ (Table 1). Among these, 5,185 corresponded to predicted gene models, including pseudogenes and gene fragments [33]. This represents $71.4 \%$ of the 7,264 predicted gene models. The genes contained on average 4.6 exons, ranging from one to 53 , which is similar to what was found in B. distachyon (5.2), rice (3.8), maize (4.1), sorghum (4.3), and Triticum urartu (4.7) [8,21,34-36]. The percentage of expressed genes is slightly lower than the ones reported in other plant species. Indeed, a microarray analysis of the rice 
Table 1 General features of the chromosome 3B pseudomolecule transcriptionally active region

\begin{tabular}{lll}
\hline Transcriptionally active regions & Predicted $^{\mathbf{a}}$ & Expressed \\
\hline Protein-coding genes & & \\
$\quad$ Total & 7,264 & 5,185 \\
$\quad$ Full genes & 5,326 & 4,125 \\
$\quad$ Pseudogenes and fragments & 1,938 & 1,060 \\
Novel transcribed regions & & \\
$\quad$ Total & - & 3,692 \\
$\quad$ Putative lincRNAs & - & 1,922 \\
Cis-NATs & & 635 \\
\hline
\end{tabular}

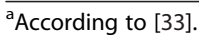

transcriptome performed in seedling shoots, tilleringstage shoots and roots, heading, filling-stage panicles, and suspension-cultured cells detected expression for $86 \%$ of the 41,754 known and predicted gene models present on the microarray [37]. More recently, Lu et al. [38] conducted an RNA-seq analysis on seeds from three rice cultivated subspecies and found that $83.1 \%$ of the 46,472 annotated gene models were expressed. Similarly, in maize, microarray-based transcript profiling in 60 distinct tissues representing 11 major organ systems revealed that $91.4 \%$ of the genes were expressed in at least one tissue [39]. More recently, Sekhon and colleagues [40] performed RNA-seq experiments on a subset of 18 selected tissues representing five organs and showed that $74.7 \%$ of the 39,429 genes from the filtered gene set were transcribed. In soybean RNA-seq analysis revealed that $80.4 \%$ of 69,145 putative genes are expressed in a least one of the 14 tissues analyzed [41]. The lower percentage of genes expressed in wheat might suggest a small impact of polyploidization on gene silencing. This is consistent with previous studies conducted in newly synthesized polyploid wheat and rapeseed where $7.7 \%$ and $4.1 \%$ of the sequences showed alteration in gene expression $[42,43]$. To estimate the exact extent of gene silencing in hexaploid wheat, a comparison with diploid and tetraploid progenitors would be required. However, when considering only genes likely to be functional (hereafter referred to as 'full genes'), the percentage of expressed genes rose to $77.5 \%(4,125 / 5,236)$, which is similar to the percentages found in maize and soybean using a similar number of conditions [40,41]. Beside full genes, $54.7 \%(1,060 / 1,938)$ regions annotated as pseudogenes or gene fragments in the pseudomolecule were found to be expressed in at least one condition. In other species such as $A$. thaliana and rice, EST analyses revealed expression for $2 \%$ to $5 \%$ and $2 \%$ to $3 \%$ pseudogenes, respectively [44]. Another study conducted on 1,439 rice pseudogenes using Massively Parallel Signature Sequencing tags suggested that up to $12 \%$ are expressed in at least one of the 22 samples studied [45]. These proportions strongly differ from our results. One cannot exclude that the percentage of pseudogenes expressed on chromosome $3 \mathrm{~B}$ could be overestimated as a result of the RNA-seq technology that cannot completely discriminate pseudogene expression from close functional copies that might be present elsewhere in the genome. In an attempt to assess this overestimation, we searched the recently released draft assembly of the wheat genome [31] for additional copies of pseudogenes in the genome. Overall, 511 out of 1,060 (48.2\%) had at least one other copy, whereas $51.8 \%$ were found to be present in one single copy located on chromosome $3 \mathrm{~B}$. Assuming that for the $48.2 \%$ 'multicopy' pseudogenes, transcripts were not produced by the $3 \mathrm{~B}$ loci, our results suggest that $28 \%$ of the chromosome $3 \mathrm{~B}$ pseudogenes are still expressed, which is much higher than what has been observed in other organisms so far. Transposable elements (TEs) have been shown to be able to generate sense or antisense transcripts of adjacent genes [46]. Given the high proportion (>85\%) of the wheat genome covered by TEs, one can hypothesize that some TEs provide a promoter for transcription of adjacent pseudogenes. In addition, while they have long been considered as non-functional units, several studies suggest that pseudogenes might play a role in regulation through antisense regulation of their parental gene, competition for miRNA, generation of small-interfering RNA, or production of short proteins or peptides $[47,48]$. The high percentage of expressed pseudogenes found in wheat compared to other species might therefore be due to their role in the regulation of homoeologous or paralogous gene expression.

For $28.6 \%$ of the predicted gene models $(2,079)$, we failed to detect any expression. This result probably reflects the fact that these genes might be expressed in specific conditions that have not been studied in the present work. Indeed, a Gene Ontology term analysis of these non-expressed genes revealed enrichment in biological processes such as 'gametophyte development', 'response to temperature stimulus', or 'response to water; (Additional file 1: Table S1). In addition, $57.5 \%$ of the non-expressed genes are non-syntenic with $B$. distachyon, rice, and sorghum, suggesting that some of these genes might have been duplicated and translocated without their regulatory sequences, leading to their transcriptional inactivity. Finally, it is worth noting that the proportion of non-expressed pseudogenes is twice as high as the proportion of non-expressed functional genes (45.3\% vs. $22.6 \%)$. As a result, the distribution pattern of non-expressed genes along the chromosome was found to be highly correlated with that of pseudogenes $\left(\mathrm{r}_{S}=0.81, P<2.2 \mathrm{e} 10^{-16}\right)$, and even more with that of single copy pseudogenes $\left(\mathrm{r}_{\mathrm{S}}=0.86, P<2.2 \mathrm{e} 10^{-16}\right)$. 
In addition to the predicted gene models, expression was detected for 3,692 loci in unannotated regions. These so-called novel transcribed regions (NTRs) represented on average $22 \%$ of all TARs. Twenty-eight percent $(1,033 / 3,692)$ of these NTR-translated sequences shared weak similarity with plant proteins, mainly TEencoded proteins or hypothetical proteins and might therefore be protein-coding genes (or pseudogenes). Out of the 2,659 with no similarity with plant proteins, 596 were longer than $200 \mathrm{nt}$ and did not carry ORFs longer than 300 AA. These NTRs might therefore correspond to long intergenic non-coding RNAs (lincRNAs) as defined by Liu and colleagues [49]. Based on this number, one could speculate that roughly 10,000 lincRNAs should be expressed in the whole wheat genome, or 3,300 per diploid genome. This number is comparable to that of expressed lincRNAs reported A. thaliana [49] and poplar [50] (2,708 and 2,542, respectively). Out of these 596 putative lincRNAs, $91.1 \%$ and $93 \%$ were found in the Triticum urartu and Aegilops tauschii genomes, respectively. The percentage decreases to $69.3 \%$ when looking at the barley genome. An even more drastic drop was observed when moving out of the Triticeae tribe, with only $14.8 \%, 7 \%$, and $6.2 \%$ of the putative lincRNAs conserved in the $B$. distachyon, rice, and sorghum genomes, respectively. These findings suggest that most of these putative lincRNAs are functional elements that have been acquired by the wheat and more largely the Triticeae genomes in the time course of their evolution.

Beside lincRNAs that are located in intergenic regions and therefore do not overlap with protein-coding genes, cis-natural antisense transcripts (NATs) are another form of long non-coding RNAs [51-53]. To estimate the extent of cis-NATs in wheat, oriented RNA-seq libraries from the five organs were constructed and reads were mapped on chromosome $3 \mathrm{~B}$ without allowing mismatches. Out of the 5,185 expressed genes, 635 (12.2\%) were found to be transcribed on the reverse strand as well, therefore producing a cis-NAT. It is worth noting that cis-NATs originate preferentially from syntenic genes $(72.4 \%)$ and the vast majority $(84.9 \%)$ concerned full genes. A previous study conducted in wheat using microarray identified 110 NATs at the whole genome level [54]. Conversely, Serial Analysis of Gene Expression showed that up to $25.7 \%$ of wheat was represented by reverse tags [55]. Such widespread occurrence of antisense transcription has already been reported in other plant species such as $A$. thaliana, rice or maize where $2.8 \%$ to $9.7 \%$ of genes produce antisense transcripts [53,56,57]. Cis-NATs can regulate gene expression at the transcriptional or post-transcriptional level through various mechanisms [51,58]. In a polyploid species, one can hypothesize that they play a role in the regulation of homoeologous copies.

\section{Transcription sites are distributed throughout the entire chromosome 3B}

The distribution of predicted protein-coding genes, nonexpressed genes, expressed genes, NTRs, and cis-NATs density was analyzed along chromosome 3B (Figure 1A). Recently, Choulet et al. [33] reported on the structural and functional partitioning of chromosome $3 \mathrm{~B}$ based on recombination pattern. While the $\mathrm{R} 1$ and $\mathrm{R} 3$ regions tended to be quite homogeneous, the R2 region appeared to be highly heterogeneous in terms of transposable element and gene content, expression breath as well as linkage disequilibrium. This is especially true in the so-called centromeric-pericentrometic region. Thus to refine our analysis of chromosome $3 \mathrm{~B}$, we divided the chromosome in five regions: R1 (1 to $68 \mathrm{Mb}$ ), R2a (68 to $265 \mathrm{Mb}$ ), C (265 to $387 \mathrm{Mb}$ ), R2b (387 to $715 \mathrm{Mb}$ ), and R3 (715 to $774 \mathrm{Mb}$ ) (Figure 1B). The densities of predicted protein-coding genes, non-expressed genes, expressed genes, NTRs, and cis-NATs were then computed in each of these regions. The density of expressed genes was highly correlated with the distance to the centromere $\left(\mathrm{r}_{s}=0.77, P<2.2 \mathrm{e}-16\right)$ and was found to follow that of predicted protein-coding genes $\left(X^{2}\right.$ test $=415.84$, $\mathrm{df}=762$ ). The overall average density was $6.5 \pm 3.3$ genes/ $\mathrm{Mb}$, ranging from 1.0 in the centromeric region up to 18.2 at the most telomeric end of the short arm. With an average density of $4.8 \pm 4.1$ per Mb, NTRs were slightly less abundant than expressed protein-coding genes but followed the overall gene distribution. However, their proportion was found to be much higher in the pericentromeric $C$ region. Since this region corresponds to the part of the chromosome where the TE density is the highest, this suggests that some of these NTRs might actually be transcribed from adjacent TE promoters. Whether these RNAs are 'transcriptional' noise or have a biological function remains to be investigated. The distribution of cis-NATs is slightly more even along the chromosome, suggesting that proximal genes are more prone to antisense transcription than distal ones. Once again, this might be due to the high abundance of TEs in these regions that would provide promoters for the transcription of adjacent genes.

Taken together, these results clearly demonstrate that transcription occurs all along chromosome $3 \mathrm{~B}$ and is not restricted to distal regions. This is in complete agreement with our previous analyses using microarray hybridizations of BAC pools and mRNA samples [29] and with observations from Abranches et al. [59] who demonstrated that active transcription sites are distributed throughout the wheat genome and do not show any preferential localization in the nuclei. More recently, Baker et al. [60] provided evidences that genes located in the low recombining pericentromeric regions were expressed at a level that was similar to that of genes in 


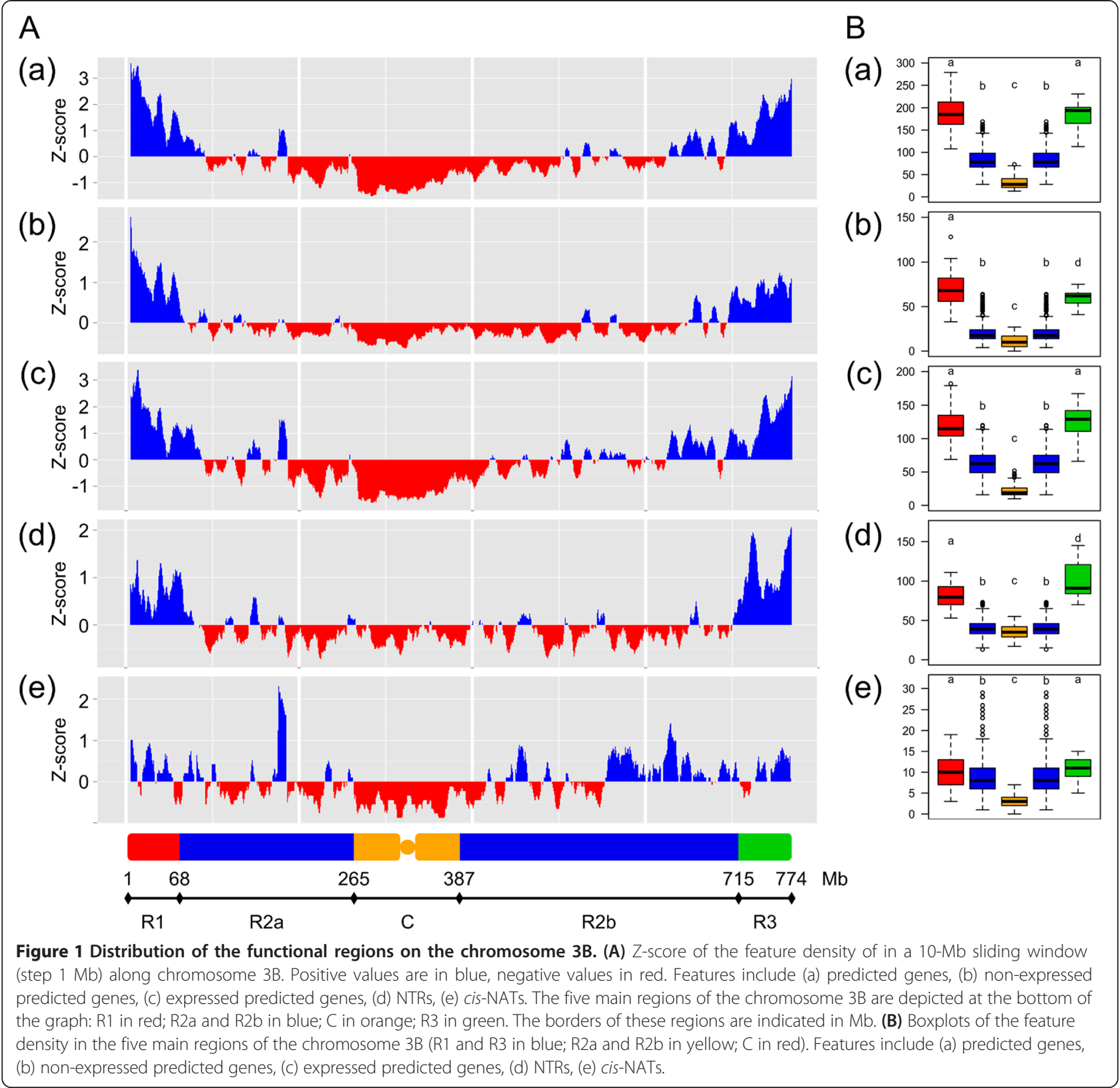

high recombining distal regions in barley. Thus, while gene density follows an increasing gradient along the centromere-telomere axis that correlates with recombination, this distribution does not seem to relate to the overall transcription capacities of wheat genes.

\section{Expression level, expression breadth, and alternative splicing are correlated}

The number of expressed genes was found to be comparable across the 15 conditions, with on average $3,734 \pm 228$ genes expressed per condition. A similar trend was observed in other species such as maize [39], soybean [41], or peach [61]. The average expression breadth (that is, the number of conditions in which a gene is expressed) for the 5,185 expressed gene models was 10.8 , with $46.2 \%(2,396)$ of the genes expressed in all conditions and $7.6 \%$ (396) exhibiting a condition-specific expression profile. At the organ level, the number of organ-specific genes ranged from 77 in leaf to 243 in spike. These proportions of condition-specific genes are not similar for all types of genes (Figure 2). For example, pseudogenes and gene fragments were found to be more specific than full genes with only $36.9 \%$ of them being expressed in 15 conditions and $10.7 \%$ in one single condition ( $v s .48 .6 \%$ and $6.9 \%$ for full genes, respectively). A similar trend was observed when comparing syntenic and 


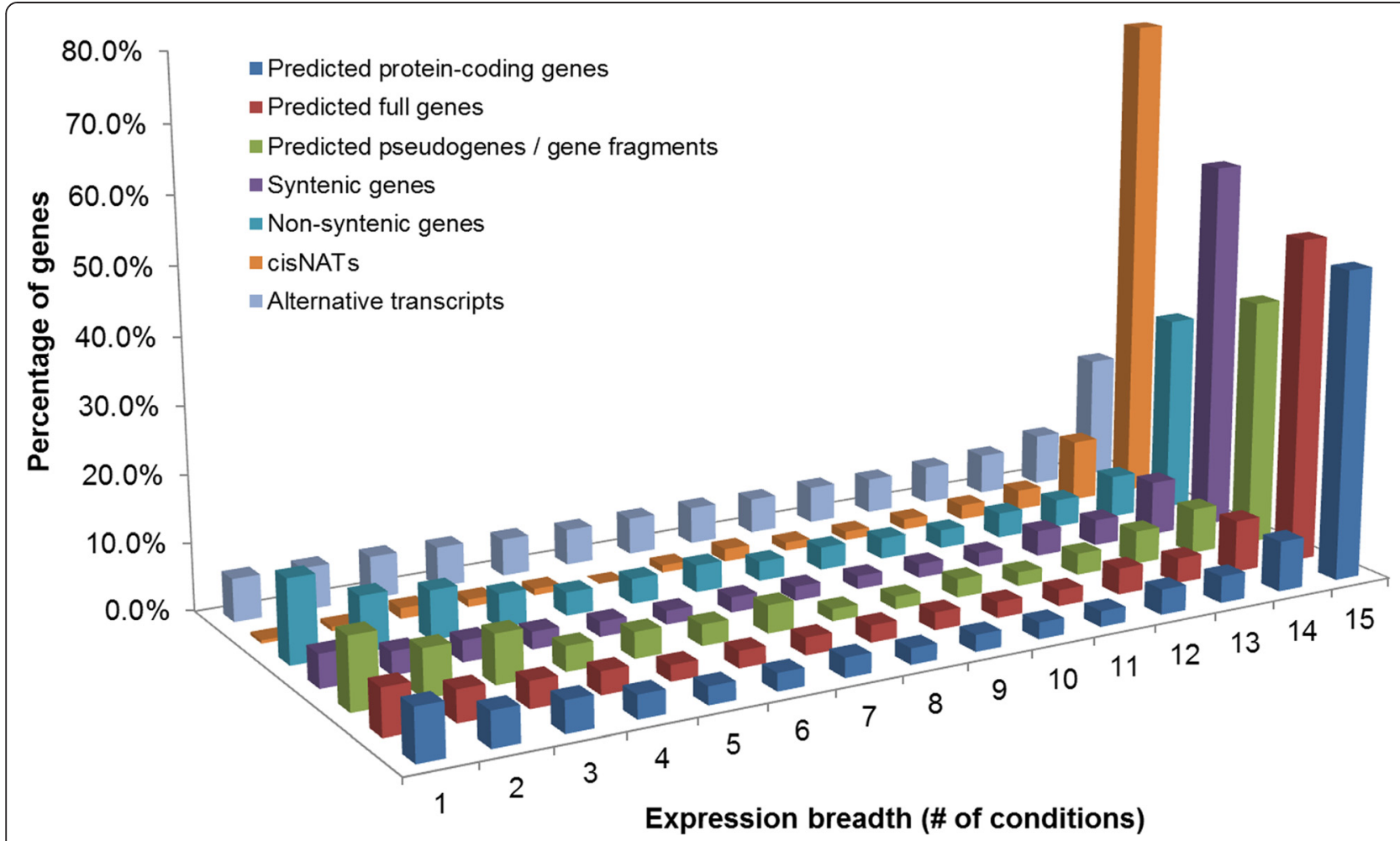

Figure 2 Distribution of the percentage of transcriptionally active regions expressed in the different number of experimental conditions. Regions were classified according to their expression breadth, that is, the number of conditions in which they were expressed, from 1 to 15. Dark blue: predicted protein-coding genes; red: predicted full genes; green: predicted pseudogenes/gene fragments; purple: syntenic genes; cyan: non-syntenic genes; orange: cis-NATs; light blue: alternative transcripts.

non-syntenic genes that were identified by comparative analysis along the 3B sequence [33]. Indeed, $29.6 \%$ of the non-syntenic genes were expressed in 15 conditions and $12.5 \%$ in one single condition, whereas $55.5 \%$ and $4.9 \%$ of syntenic genes were found to be expressed in 15 and one condition, respectively. By contrast, $73.7 \%$ of genes showing anti-sense transcription were expressed in 15 conditions while only very few of them $(0.5 \%)$ were specific to one single condition. This reinforces the idea that cisNATs serve as post-transcriptional regulators of gene expression [62-64].

Expression breadth was found to be correlated with expression level. This correlation is not unexpected since genes that are widely expressed such as house-keeping genes tend to show a higher expression level than condition-specific genes $[17,65,66]$. However, to some extent, one cannot exclude that this relationship between expression level and expression breadth reflects the fact that expression is not detected in some conditions and that some condition-specific genes might just be low expression genes.

Our analysis revealed 30,232 transcripts originating from the 5,185 chromosome 3B expressed genes. Thirtynine percent of the genes were transcribed in one single
mRNA in our conditions whereas splicing variants were detected for $61.4 \%$, with an average of 5.8 alternative transcripts per gene. When considering multiexonic genes only, the percentage of alternatively spliced genes raised to $75.4 \%$. While alternative splicing (AS) is a general phenomenon in plants, the overall AS level differs strikingly between species. Indeed, previous studies reported that $61 \%$ and $48 \%$ of $A$. thaliana and rice genes undergo AS, respectively $[38,67]$, whereas only $6.3 \%$ and $15.9 \%$ of expressed genes are under the potential influence of AS in B. distachyon and soybean, respectively $[68,69]$. In barley, $55 \%$ of high confidence genes and $73 \%$ of intron-containing high confidence genes have evidence of AS [70]. This high similarity between barley and wheat, as well as differences with that of rice and $B$. distachyon suggests that the AS level might have evolved differently in grasses. Conversely, considering that the level of AS observed in wheat was similar to that of A. thaliana, it is very unlikely that these differences between species are linked to genome size and complexity. However, one cannot exclude that differences originate from experimental design. In $A$. thaliana, the predicted AS level increased from 1.2\% to $61 \%$ between 2003 and 2012, mainly as a result of the advent 
of high-throughput technologies [71]. In addition, alternative transcripts have been hypothesized to be tissueor condition-specific [72]. As our results are based on the study of plants grown in normal conditions we cannot exclude that the percentage of AS genes is underestimated and might increase with the inclusion of other samples such as plants grown under stress conditions.

Beside the differences observed in the overall AS level between species, we found differences in the relative abundance of the main types of AS, namely exon skipping (ES), alternative splice sites (A3SS and A5SS), intron retention (IR), and mutually exclusive exons (MXE) [73]. In wheat, IR was found to be the predominant type, with $35 \%$ of all events, followed by A3SS (27\%), ES (21\%), A5SS (16\%), and MXE (0.9\%). In A. thaliana, rice, and $B$. distachyon, IR accounts for more than 50\% $[68,74]$ whereas the predominant type was found to be ES in the peach genome, with $43 \%$ of all observed events [61]. Such differences strongly reinforce the idea that, despite the fact that AS is a common phenomenon shared by most if not all plant species, specificities have been acquired by the different plant species during the course of their evolution.

While $46.2 \%$ of the 5,185 genes were expressed in 15 conditions, only $18.6 \%$ of 30,232 transcripts appeared to be present in all conditions, which is very similar to what has been observed in barley [70]. In addition, 95\% of the AS transcripts originating from the same gene exhibited different expression profiles, as revealed by a hierarchical clustering of the 30,232 transcripts (data not shown). As a consequence, the number of alternative transcripts was found to be positively correlated with the expression breadth. These findings strongly suggest that AS variants have complementary functions across organs or developmental stages.

\section{A non-monotonic relationship between gene expression and gene structure}

A negative correlation was observed between the transcript size and the expression breadth, with shorter transcripts being expressed in more conditions. This is consistent with previous studies indicating that housekeeping genes which are expressed in more conditions are generally more compact than genes expressed in specific conditions $[17,65,66,75]$. Such findings could be explained by the 'selection for economy' model $[17,76]$. In this model transcription and translation are both timeand energy-consuming and, as a consequence, widely and highly expressed genes tend to be more compact to reduce the energetic cost $[77,78]$.

We then investigated the correlation between expression level and gene structure, in terms of transcript size, number of exons, cumulative intron length, mean exon and intron length, and number of alternative transcripts.
To this aim, genes were grouped in 30 classes of similar size based on their average expression level (that is, the average FPKM value in a number of conditions where the gene is expressed), as done by Carmel and Koonin [16]. Then, the average values of different variables in each of the 30 expression level classes were computed across the genes (Figure 3).

For the transcript size, the number of exons, the cumulative intron length, and the number of alternative transcripts, non-monotonic relationships were found with the expression level, resulting in an approximate bellshaped dependence (Figure 3). For all features, the area of the inflexion point was comprised between classes 20 and 25 which is also the area where expression breadth reaches a plateau. Following criteria defined by Hansey et al. [79], genes in categories 1 to 4 correspond to low expression genes (mean expression level <5 FPKM), those in categories 5 to 21 to medium expression genes ( $5 \leq$ mean expression level $<200$ FPKM) and those in categories 22 to 30, to high expression genes (mean expression level $\geq 200$ FPKM). Interestingly the inflexion point also corresponded to the threshold between medium and high expression genes. For medium expression genes, the expression level was positively correlated with the structural features whereas for high expression genes, we found a negative correlation. For low expression genes, the observed relationship might be an artifact resulting from the detection threshold of low abundance transcripts. Indeed some of these genes might have been considered as expressed when actually they were not. Therefore, these four classes (1 to 4) might not be reliable as they might contain a mix of expressed and nonexpressed genes leading to average structural feature values that are not representative of expressed genes. For the mean exon and intron length, no clear relationship was observed even though the mean exon length tends to decrease as the expression level increases. Such a nonmonotonic relationship has already been observed in other organisms, including human, Caenorhabditis elegans, Drosophila melanogaster, A. thaliana, and soybean $[16,18]$. If the 'selection for economy' fits for highly expressed genes, it cannot apply to low to medium expression genes. The 'genome design' model has been proposed to explain this relationship $[17,66,75,76]$. It suggests that the structural features of a gene are mostly determined by its functional load. Highly and widely expressed genes would not require a fine regulation and therefore less regulatory sequences. By contrast, for low/medium expression, condition-specific genes, longer intragenic non-coding sequences would allow for a more complex regulation. Since the number of alternative transcripts follows the same distribution, one can hypothesize that the greater number of exons and the larger intronic sequences might allow for a greater transcriptional complexity leading to a greater 


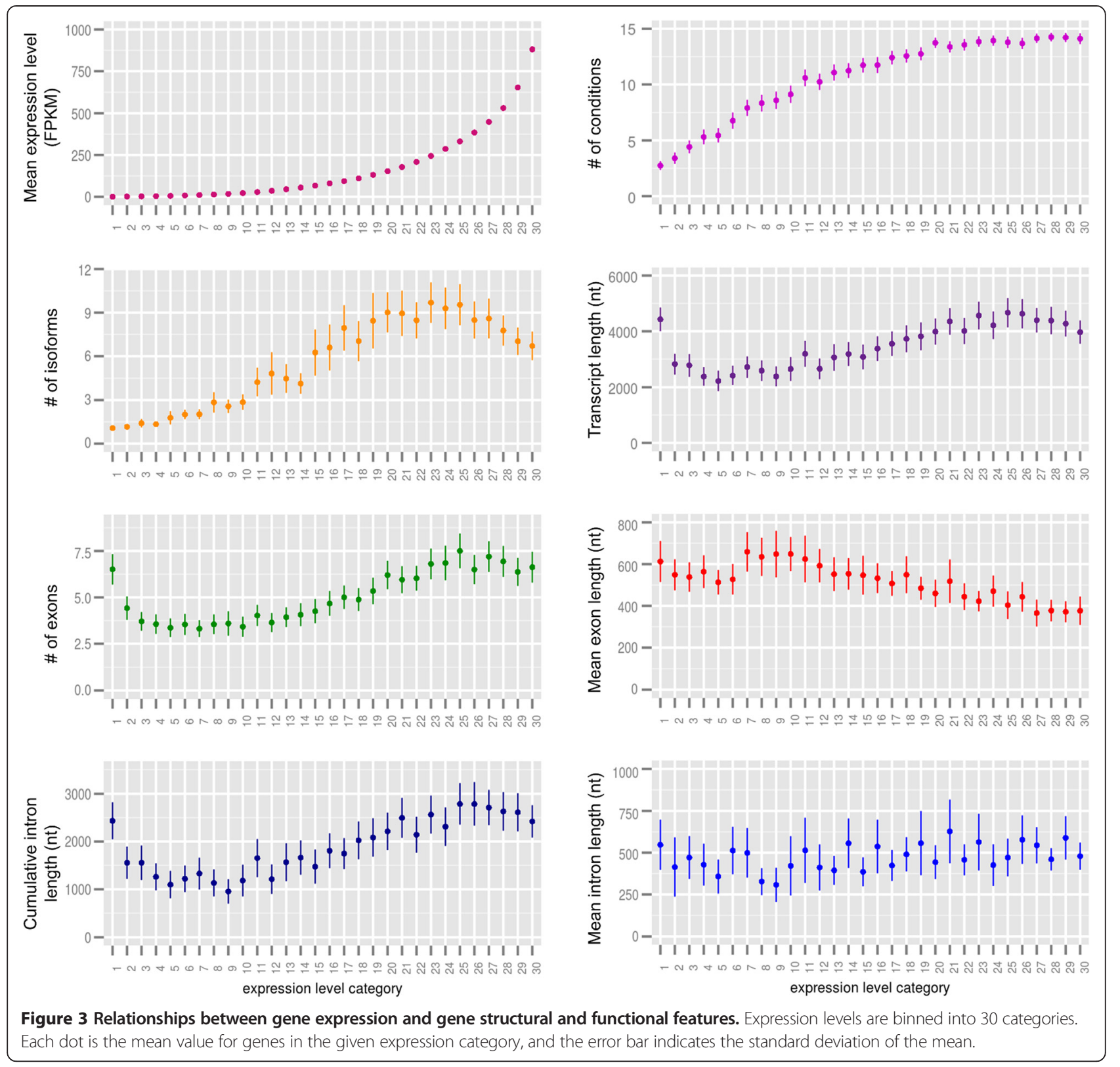

specificity in gene expression. A detailed analysis of transcript size based on the 30,232 isoforms showed a negative correlation with expression level, regardless of the expression class (Additional file 2: Figure S1). This finding reinforces the hypothesis that gene structure would be determined by its function ('genome design' model) whereas the expression of the different transcripts from a given gene would be subject to the energetic cost ('selection for economy' model).

\section{Gene structural and functional features are partitioned on chromosome 3B}

To investigate their relationship with chromosome partitioning, the expression breadth, expression level, transcript size, number of exons, cumulative intron length, mean exon and intron length, and the number of alternative transcripts were computed in the five regions of chromosome 3B, namely R1, R2a, C, R2b, and R3 (Figure 4A). For all features but mean exon and intron length, the regions can be classified in three contrasting groups. The first one includes regions R1 and R3, the second one, R2a and R2b, and the third one, $\mathrm{C}$. All of the features decrease along the centromere-telomere axis. Thus, on average, genes in distal regions are expressed at a lower level, more specifically and have fewer isoforms than those in the proximal regions. In addition, they are shorter, with fewer exons and shorter intronic sequences. Genes located in region $\mathrm{C}$ tend to have shorter exons, while for the mean intron length, no 


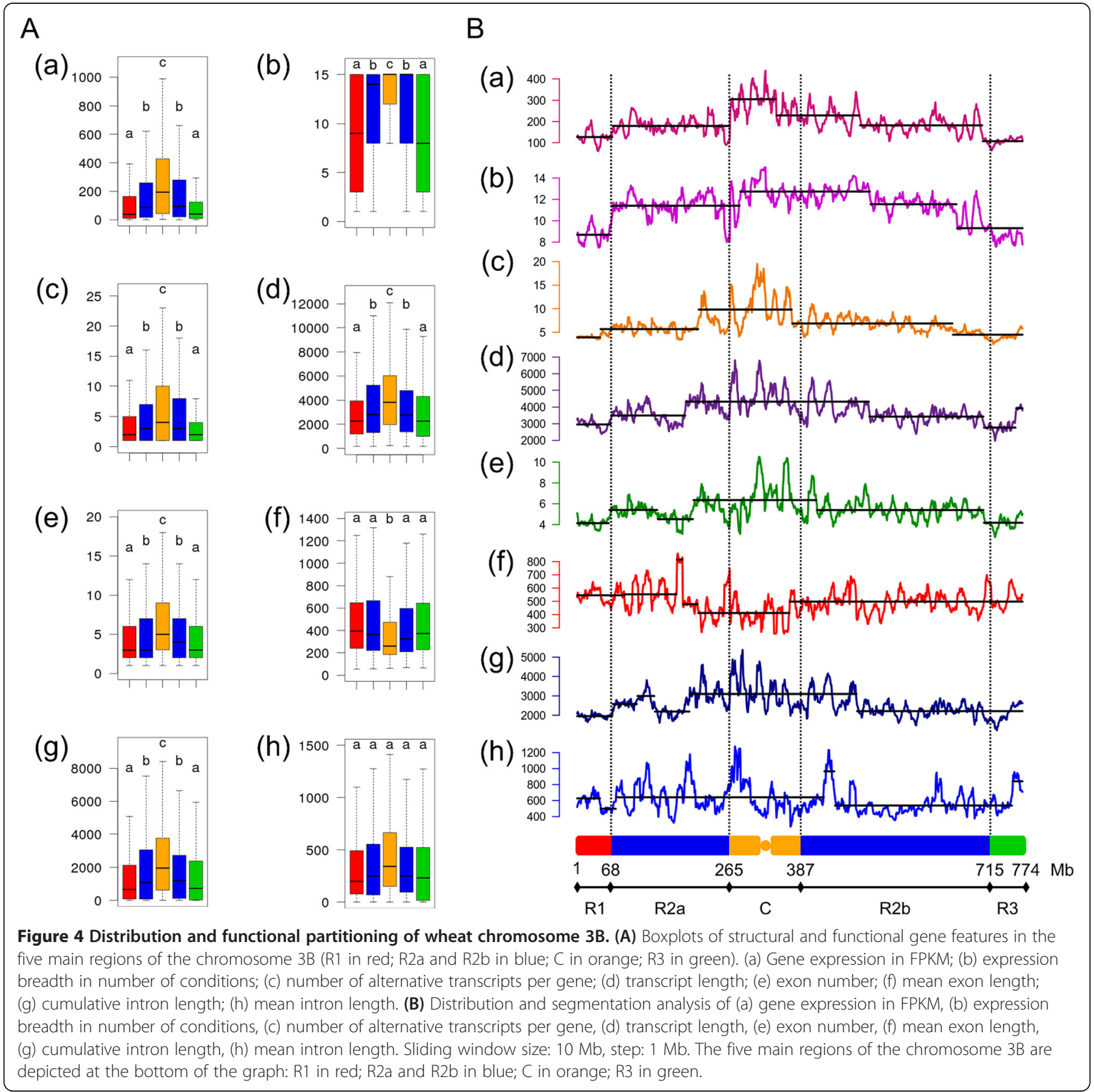

significant differences were observed between the five regions. A segmentation analysis of these properties suggests a partitioning of the chromosome rather than a regular gradient from centromere to telomeres (Figure 4B). Interestingly, the boundaries of the distal segments fit almost perfectly with the R1 and R3 regions defined by Choulet et al. [33] based on recombination. It is worth noting that the analysis of chromosomes $3 \mathrm{~A}$ and 3D based on their draft assembly [31] also revealed a strong partitioning of expression breadth, suggesting that the features observed on chromosome $3 \mathrm{~B}$ should be conserved at the whole wheat genome level (Additional file 1: Figure S2).
To see to what extent the non-monotonic relationship between expression level and gene structural features observed at the whole chromosome level is conserved at the region level, we applied the same analysis to regions $\mathrm{R} 1 / \mathrm{R} 3$ and $\mathrm{R} 2 \mathrm{a} / \mathrm{R} 2 \mathrm{~b}$. Region $\mathrm{C}$ was not included due to the limited number of genes present in this region. Interestingly, the chromosomal pattern remained the same in each region (Additional file 1: Figures S3 and S4). Even though the average expression level was lower in R1/R3 regions, the mean expression level value of the inflexion point was conserved in the two regions, around 200 FPKM, the approximate threshold between 
medium and high expression genes. This finding clearly shows that the 'selection for economy' and 'genome design' models apply all along the chromosome independently of other features and strongly suggests that the evolutionary forces that have led to the chromosome partitioning are distinct from the molecular mechanisms governing gene expression.

\section{Chromosome conformation may play a role in gene regulation}

A hierarchical clustering of the 5,185 expressed proteincoding gene primary transcripts was performed based on their expression profiles in the 15 conditions. Genes were aggregated into 55 distinct clusters according to their expression profiles. Based on the median value of intergenic distances of $30 \mathrm{~kb}$, Choulet et al. [33] estimated that $73 \%$ of genes were organized in small islands or 'insulae'. Using the same criteria, 3,465 out of the 5,185 expressed genes (67\%) were found to be organized in 1,199 insulae, comprising 2.9 genes on average. Out of these 3,465 genes, 1,218 (35.2\%) belong to the same expression cluster as their direct neighbor, defining 718 co-expressed gene pairs. This proportion is higher than the previously reported value of $11 \%$ [29] most probably because of the higher resolution achieved with a reference sequence compared to a partial gene dataset. Such enrichment has already been reported in other organisms such as human, mouse, A. thaliana, rice, and fruit fly where the percentage of adjacent co-expressed genes is in the range of $2 \%$ to $20 \%[11,12,15,80,81]$. However, these percentages are relatively low compared to that of wheat. One can hypothesize that the higher proportion of co-expressed genes found in wheat might result from the high rate of tandem duplication in this genome [33]. However, of the 718 co-expressed gene pairs, only 46 (6.4\%) correspond to duplicated genes. This clearly shows that duplicate genes alone do not explain the observed levels of co-expression, as already reported in other organisms $[9,13,15,82]$. Several other mechanisms have been proposed to explain the co-expression of neighboring genes, including shared promoters and chromatin remodeling. In A. thaliana, Chen et al. [82] showed that co-expression was strongly enhanced for divergently transcribed genes within a 400-bp gene distance, probably as a result of shared promoters. For longer intergenic distances, co-expression is likely mediated by shared chromatin environments. On chromosome 3B, the average intergenic distance between co-expressed neighbor genes is $6.3 \mathrm{~kb}$ and only 133 out of the 718 gene pairs are transcribed divergently. This suggests that shared promoters are not the main mechanism controlling the co-expression of neighbor genes and that other mechanisms such as chromatin conformation might be involved. This hypothesis is reinforced by the significant differences observed for 23 out of the 55 expression clusters between the five regions (Additional file 1: Table S2). For example, the vast majority (63\%) of the genes present in the region $\mathrm{C}$ belong to cluster I that correspond to genes expressed in all conditions whereas this cluster represents only $23 \%$ to $24 \%$ of region R 1 and R3 genes (Figure 5). Region R1 is enriched in genes preferentially expressed in leaf compared to other regions. Region R3 displays a higher proportion of spikeand grain-specific genes. In addition, expression clusters oscillated along chromosome, forming chromosomal domains. These findings are consistent with the Gene Ontology term enrichment analysis that revealed that distal regions were enriched in genes involved in adaptive processes such as response to abiotic stimuli or stress [33].

Even though transcription sites are distributed throughout the entire chromosome when looking at the plant development at a whole, our results show that $3 \mathrm{~B}$ is organized in chromosomal domains, suggesting that gene position influences the spatio-temporal regulation of their expression. Such domains have recently been reported for genes expressed in wheat endosperm [23]. While no overall subgenome dominance has been observed in wheat, abundant transcriptional dominance of subgenome segments as well as asymmetrical expression of neighboring genes were observed [23,31]. This strongly differs from other polyploid or paleopolyploid species such as cotton, Brassica rapa or maize in which a clear subgenome dominance was observed [83-85]. This specific pattern of the wheat genome suggests that, in this species, polyploidization might have impacted gene expression through the formation of dominant chromosome domains rather than overall subgenome dominance. In addition, it has been shown that the spatial organization of genomes in the interphase cell nucleus is tissue-specific [86]. This positioning of chromosomes is non-random and is likely to play a role in gene regulation $[87,88]$. In wheat, the interphase chromosomes are not fully decondensed but adopt a regular Rabl configuration, a highly polarized pattern with the two chromosome arms lying next to each other and the centromeres and telomeres located at opposite poles of the nuclei [89-91]. The presence of this organization is also known to vary greatly among tissues or developmental stages of an organism [90]. Then, one can hypothesize that this configuration might play a role in gene regulation through the partial decondensation of given chromosomal regions in specific tissues and at specific developmental stages, leading to the observed spatial partitioning of genes displaying similar expression profiles. This hypothesis is well supported by our previous results [33]. Indeed, a similar recombination- and expression breadth-based partitioning was found in barley in which the Rabl configuration is also observed, but not in maize which displays 


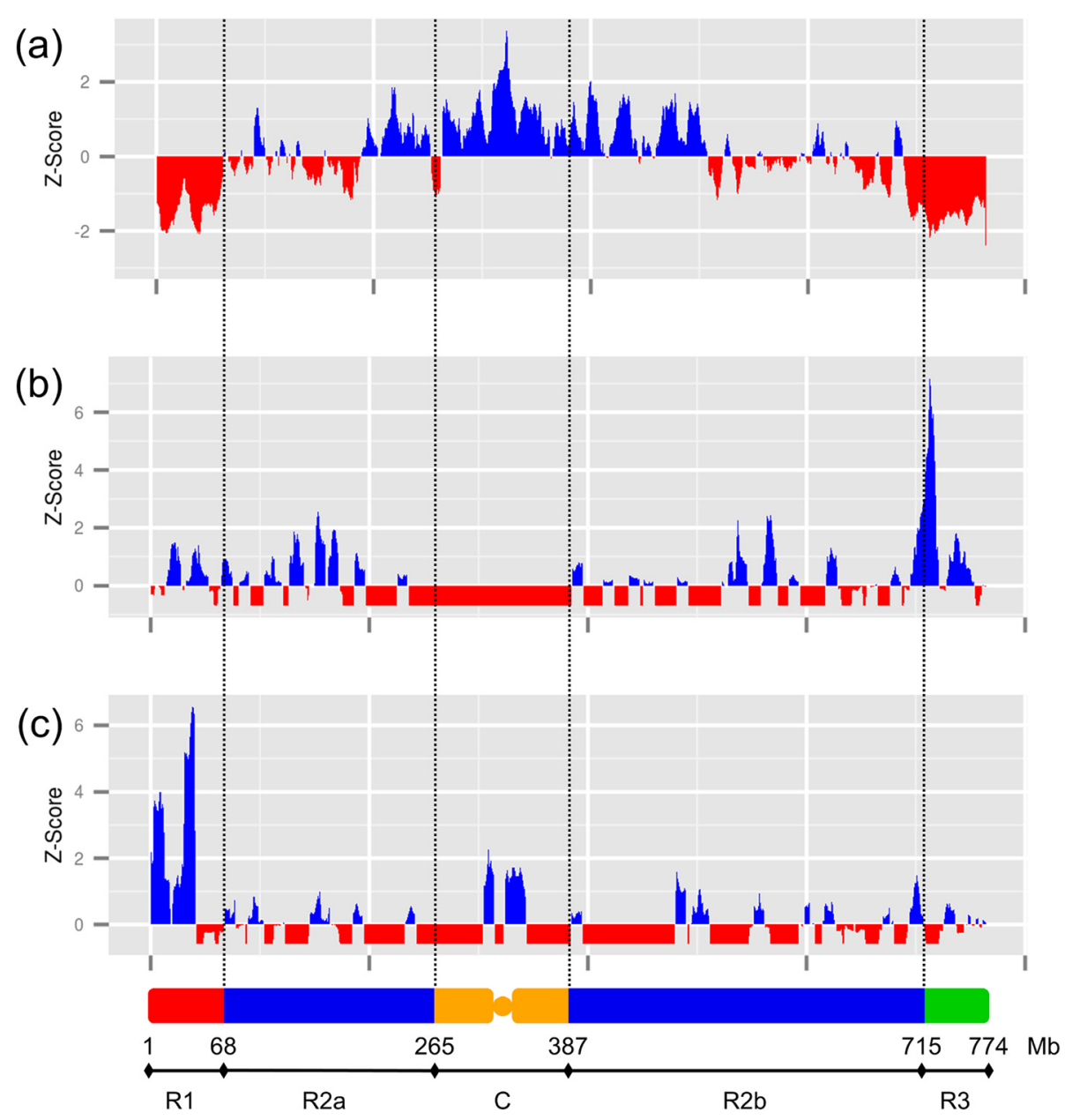

Figure 5 Distribution of the percentage of genes from three different expression clusters. Z-score of the percentage of expressed genes for a given cluster in a 10-Mb sliding window (step $1 \mathrm{Mb}$ ) along chromosome 3B. Positive values are in blue, negative values in red. (a) Constitutively expressed genes; (b) spike- and grain-specific genes; (c) genes preferentially expressed in leaf. The five main regions of the chromosome 3B are depicted at the bottom of the graph: R1 in red; R2a and R2b in blue; C in orange; R3 in green.

neither entirely Rabl nor entirely random chromosome organization $[89,90]$.

\section{Conclusions}

By combining the first reference sequence of a wheat chromosome with deep transcriptome sequencing data covering the whole plant development, we constructed a high density transcription map of the wheat chromosome $3 \mathrm{~B}$, comprising more than 8,800 transcriptionally active regions distributed throughout the entire chromosome. By studying the relationships between genome and gene structure and expression, we unraveled two interconnected mechanisms. The first one is a universal mechanism that relates to the 'selection for economy' and 'genome design' models and links gene structure and function, regardless of the gene position. The second one is an evolutionary force that links gene structure and function to gene position, leading to a strong partitioning of the wheat chromosome 3B. Since this partitioning is also observed in barley but not in other grasses, one can hypothesize that it has evolved with genome organization and is related to Triticeae-specific adaptation.

\section{Material and methods}

\section{Sample preparation and sequencing}

Total RNAs were extracted in duplicates from five organs (root, leaf, stem, spike, and grain) at three developmental stages each from hexaploid wheat $c v$. Chinese Spring [28] (Additional file 1: Table S3). RNA quality was assessed using an RNA nano Chip on the Agilent 2100 Bioanalyzer (Agilent Technologies, Santa Clara, CA, USA) and the RNA integrity number (RIN) was calculated for each sample. Only sample with a RIN greater than 7 were used for the library construction. 
The 30 strand-non-specific RNA-seq libraries (representing the 15 conditions in duplicates) were constructed from $4 \mu \mathrm{g}$ of total RNA using the Illumina TruSeq ${ }^{\mathrm{Tm}}$ RNA sample preparation Kit (Illumina, San Diego, CA, USA) according to the manufacturer's protocol, with a library insert size of $300 \mathrm{bp}$ (fragmentation time of $12 \mathrm{~min}$ ). Library profiles were evaluated using an Agilent 2100 Bioanalyzer. Illumina indexes were used to pool two samples per lane. Libraries were sequenced on an Illumina HiSeq2000 with $2 \times 100$-bp paired-end reads.

For strand-specific RNA-seq libraries, $12 \mu \mathrm{g}$ of total RNAs from the same organ were pooled $(4 \mu \mathrm{g}$ per developmental stage) and polyA+ enriched RNAs were purified using the Ambion MicropolyA Purist Kit (Life Technologies, Carlsbad, CA, USA). Fifty nanograms of purified poly A+ RNAs were used to construct the oriented RNA-seq libraries with the ScriptSeq v2 RNA-seq Library Preparation kit (Epicentre, Madison, WI, USA) following the manufacturer's instructions. After cDNA synthesis, 15 cycles PCR were performed to amplify the fragments. Libraries were purified by Ampure beads (Beckmann Coulter, Indianapolis, IN, USA) and then quantified using a Qubit Fluorometer (Life Technologies). Library profiles were evaluated using an Agilent 2100 Bioanalyzer. Each library was sequenced using 101 base-length read chemistry on one lane of a single-end (SE) flow cell on the Illumina HiSeq2000. Read quality was checked with the FastQC v0.10.0 software [92]. RNA-Seq data have been deposited under accession number ERP004714.

\section{Read alignment and expression analysis}

Illumina reads were mapped on the chromosome $3 \mathrm{~B}$ scaffolds using Tophat2 v2.0.8 [93,94] and bowtie2 [95] with the default parameters except: 0 mismatch, 0 splice-mismatch. PCR duplicates were removed with Samtools [96] rmdup option and an annotation-guided read alignment was performed with Cufflinks v2.1.1 $[93,97]$ to reconstruct transcripts and estimate transcript abundance in units of fragments per $\mathrm{kb}$ of exon per million mapped reads (FPKM) [98]. Regions with FPKM values higher than zero were considered as expressed. TriAnnotpredicted regions were distinguished from unannotated regions (novel transcribed regions, NTRs) using the -g option. NTRs were reconstructed and ORFs were detected using transcripts_to_best_scoring_ORFs.pl (Trinity) [99] and blasted against the Magnoliophyta database (BLASTX, e-value 10e-5). Based on the FPKM scale defined in by Hansey and collaborators [79] expressed genes can be divided in three classes: genes with a FPKM value below 5 are low expressed, genes with a FPKM value greater or equal to 5 and less than or equal to 200 are medium expressed, and genes with a FPKM value greater than 200 are high expressed (semi-quantitative organization).
Sequences and annotations of the reference pseudomolecule and unassigned scaffolds have been deposited in ENA (project PRJEB4376) under accession numbers HG670306 and CBUC010000001 to CBUC010001450, respectively.

\section{Segmentation/change-point analysis}

Segmentation analyses were performed using the $R$ package changepoint v1.0.6 [100] with Binary Segmentation method and BIC penalty on the mean change. The different features that were subjected to this analysis were: recombination rate, transposable element density, predicted gene density, number of condition in which a gene is expressed. All these features were calculated in sliding windows of $10 \mathrm{Mb}$ with a step of $1 \mathrm{Mb}$.

\section{Statistical analysis}

All statistical analyses were performed with the $\mathrm{R}$ software [101]. Shapiro-Wilk test was used to test for normality of distribution. Correlation analyses were performed with Spearman rank correlation method. Outlier detection was performed using the formula: (Quantile 3 Quantile 1) $\times 3$ / Quantile 3, based on FPKM value and transcripts length of each gene. Genes were classified according to their average expression level and divided in 30 classes, with the same number of genes per class. R package ggplot2 was used to draw plot. Average comparison was performed using Welsh t.test to test for statistical significance between the five regions.

\section{Hierarchical clustering}

Hierarchical clustering was performed using the Hierarchical Clustering Explorer 3.5 software [102] with the complete linkage method and the Pearson correlation coefficient. The minimal similarity to establish the clusters was set to 0.641 which is the Pearson correlation significant at the $P$ value threshold of 0.01 .

\section{Gene ontology enrichment analysis}

GOBU (Gene Ontology Browsing Utility [103]) was used for enrichment calculations. The full set of $3 \mathrm{~B}$ gene products annotated on the pseudomolecule [33] was used as the reference comparison set for the enrichment analysis of non-expressed genes. $P$ values were calculated under GOBU with the Multiview Plugin and Fisher's exact test.

\section{Additional files}

Additional file 1: Table S1. GO term enrichment analysis of non-expressed genes. Table S2. Distribution of the 5,185 expressed protein coding genes according to their expression cluster and their chromosomal region. Table S3. Wheat RNA samples used for RNA-seq experiments. 
Additional file 2: Figure S1. Alternative transcript length as a function of expression level category. Figure S2. Expression breadth-based segmentation analysis of homoeologous chromosomes 3A and 3D. Figure S3. Relationships between gene expression and gene structural and functional features in the R1 / R3 regions. Figure S4. Relationships between gene expression and gene structural and functional features in the R2a / R2b regions.

\section{Abbreviations}

A3SS: Alternative 3' splice site; A5SS: Alternative 5' splice site; AS: Alternative splicing; BLAST: Basic Local Alignment Search Tool; bp: Base pairs; cis-NAT: cisnatural antisense transcript; DNA: Deoxyribonucleic acid; FPKM: Fragments per kb of exon per million mapped reads; IR: Intron retention; kb: Kilobase pairs; lincRNA: Long intergenic non-coding ribonucleic acid; Mb: Megabase pairs; miRNA: Micro ribonucleic acid; mRNA: Messenger ribonucleic acid; MXE: Mutually exclusive exon; NAT: Natural antisense transcript; ncRNA: Noncoding ribonucleic acid; nt: Nucleotide; NTR: Novel transcribed region; ORF: Open reading frame; RNA: Ribonucleic acid; RNA-seq: Sequencing of RNA; TAR: Transcriptionally active region; TE: Transposable element.

\section{Competing interests}

The authors declare that they have no competing interests.

\section{Authors' contributions}

LP constructed RNA-seq libraries, carried out RNA-seq data analyses, and drafted the manuscript. FC participated in RNA-seq data analyses and interpretation. AA and PW produced RNA-seq data. NG performed paralogous and syntenic/non-syntenic gene analyses. CF acquired the funding and corrected the manuscript. EP designed research, supervised all the analyses and drafted the manuscript. All authors read and approved the final manuscript.

\section{Acknowledgements}

The authors would like to thank Hélène Rimbert for assistance in bioinformatics analysis. They also thank the International Wheat Genome Sequencing Consortium (IWGSC) for pre-publication access to the chromosome-based draft sequence of the wheat genome. This work was supported by grants from the French National Research Agency (ANR-09-GENM-025 3BSEQ) and France Agrimer. LP was funded by a grant from Région Auvergne.

\section{Author details}

'INRA UMR1095 Genetics, Diversity and Ecophysiology of Cereals, 5 chemin de Beaulieu, 63039 Clermont-Ferrand, France. ${ }^{2}$ University Blaise Pascal UMR1095 Genetics, Diversity and Ecophysiology of Cereals, 5 chemin de Beaulieu, 63039 Clermont-Ferrand, France. ${ }^{3}$ CEA/DSV/IG/Genoscope, 2 rue Gaston Cremieux, 91000 Evry, France. ${ }^{4}$ CNRS UMR 8030, 2 rue Gaston Crémieux, 91000 Evry, France. ${ }^{5}$ Université d'Evry, CP5706 Evry, France. ${ }^{6}$ Current address: Bayer Cropscience, Technologiepark 38, Zwijnaarde 9052, Gent, Belgium. ${ }^{7}$ Current address: Bayer CropScience, 3500 Paramount Parkway, Morrisville, NC 27560, USA.

\section{Received: 24 September 2014 Accepted: 28 January 2015}

\section{Published online: 10 February 2015}

\section{References}

1. Bennett MD, Leitch IJ. Nuclear DNA amounts in angiosperms: targets, trends and tomorrow. Ann Bot. 2011;107:467-590.

2. Ming R, Hou S, Feng Y, Yu Q, Dionne-Laporte A, Saw JH, et al. The draft genome of the transgenic tropical fruit tree papaya (Carica papaya Linnaeus). Nature. 2008;452:991-6.

3. CoGePedia. [http://genomevolution.org/wiki/index.php/Main_Page]

4. The International Brachypodium Initiative. Genome sequencing and analysis of the model grass Brachypodium distachyon. Nature. 2010;463:763-8.

5. Jaillon O, Aury JM, Noel B, Policriti A, Clepet C, Casagrande A, et al. The grapevine genome sequence suggests ancestral hexaploidization in major angiosperm phyla. Nature. 2007;449:463-7.

6. Tuskan GA, Difazio S, Jansson S, Bohlmann J, Grigoriev I, Hellsten U, et al. The genome of black cottonwood, Populus trichocarpa (Torr. \& Gray). Science. 2006;313:1596-604.

7. Schmutz J, Cannon SB, Schlueter J, Ma J, Mitros T, Nelson W, et al. Genome sequence of the palaeopolyploid soybean. Nature. 2010;463:178-83.
8. Schnable PS, Ware D, Fulton RS, Stein JC, Wei F, Pasternak S, et al. The B73 maize genome: complexity, diversity, and dynamics. Science. 2009;326:1112-5.

9. Lee JM, Sonnhammer EL. Genomic gene clustering analysis of pathways in eukaryotes. Genome Res. 2003;13:875-82.

10. Liu X, Han B. Evolutionary conservation of neighbouring gene pairs in plants. Gene. 2009;437:71-9.

11. Ren XY, Fiers MW, Stiekema WJ, Nap JP. Local coexpression domains of two to four genes in the genome of Arabidopsis. Plant Physiol. 2005;138:923-34.

12. Ren XY, Stiekema WJ, Nap JP. Local coexpression domains in the genome of rice show no microsynteny with Arabidopsis domains. Plant Mol Biol. 2007;65:205-17.

13. Williams EJ, Bowles DJ. Coexpression of neighboring genes in the genome of Arabidopsis thaliana. Genome Res. 2004;14:1060-7.

14. Xu Z, Kohel RJ, Song G, Cho J, Alabady M, Yu J, et al. Gene-rich islands for fiber development in the cotton genome. Genomics. 2008;92:173-83.

15. Zhan S, Horrocks J, Lukens LN. Islands of co-expressed neighbouring genes in Arabidopsis thaliana suggest higher-order chromosome domains. Plant $\mathrm{J}$. 2006:45:347-57.

16. Carmel L, Koonin EV. A universal nonmonotonic relationship between gene compactness and expression levels in multicellular eukaryotes. Genome Biol Evol. 2009;1:382-90.

17. Vinogradov AE. 'Genome design' model and multicellular complexity: golden middle. Nucleic Acids Res. 2006;34:5906-14.

18. Woody JL, Severin AJ, Bolon Y-T, Joseph B, Diers BW, Farmer AD, et al. Gene expression patterns are correlated with genomic and genic structure in soybean. Genome. 2011;54:10-8.

19. Neale D, Wegrzyn J, Stevens K, Zimin A, Puiu D, Crepeau M, et al. Decoding the massive genome of loblolly pine using haploid DNA and novel assembly strategies. Genome Biol. 2014;15:R59.

20. Chen ZJ, Scheffler BE, Dennis E, Triplett BA, Zhang T, Guo W, et al. Toward sequencing cotton (Gossypium) genomes. Plant Physiol. 2007;145:1303-10.

21. Ling HQ, Zhao S, Liu D, Wang J, Sun H, Zhang C, et al. Draft genome of the wheat A-genome progenitor Triticum urartu. Nature. 2013;496:87-90.

22. Jia J, Zhao S, Kong X, Li Y, Zhao G, He W, et al. Aegilops tauschii draft genome sequence reveals a gene repertoire for wheat adaptation. Nature. 2013;496:91-5

23. Pfeifer M, Kugler KG, Sandve SR, Zhan B, Rudi H, Hvidsten TR, et al. Genome interplay in the grain transcriptome of hexaploid bread wheat. Science. 2014;345:1250091.

24. Gillies SA, Futardo A, Henry RJ. Gene expression in the developing aleurone and starchy endosperm of wheat. Plant Biotechnol J. 2012;10:668-79.

25. Pellny TK, Lovegrove A, Freeman J, Tosi P, Love CG, Knox JP, et al. Cell walls of developing wheat starchy endosperm: comparison of composition and RNA-Seq transcriptome. Plant Physiol. 2012;158:612-27.

26. Bartoš J, Paux E, Kofler R, Havránková M, Kopecký D, Suchánková P, et al. A first survey of the rye (Secale cereale) genome composition through BAC end sequencing of the short arm of chromosome 1R. BMC Plant Biol. 2008;8:95.

27. Ergen NZ, Thimmapuram J, Bohnert HJ, Budak H. Transcriptome pathways unique to dehydration tolerant relatives of modern wheat. Funct Integr Genomics. 2009;9:377-96.

28. Choulet F, Wicker T, Rustenholz C, Paux E, Salse J, Leroy P, et al. Megabase level sequencing reveals contrasted organization and evolution patterns of the wheat gene and transposable element spaces. Plant Cell. 2010;22:1686-701.

29. Rustenholz C, Choulet F, Laugier C, Safár J, Simková H, Dolezel J, et al. A 3,000-loci transcription map of chromosome $3 \mathrm{~B}$ unravels the structural and functional features of gene islands in hexaploid wheat. Plant Physiol. 2011;157:1596-608.

30. Raats D, Frenkel Z, Krugman T, Dodek I, Sela H, Simkova H, et al. The physical map of wheat chromosome 1BS provides insights into its gene space organization and evolution. Genome Biol. 2013;14:R138.

31. The International Wheat Genome Sequencing Consortium. A chromosomebased draft sequence of the hexaploid bread wheat genome. Science. 2014;345:1251788.

32. Brenchley R, Spannagl M, Pfeifer M, Barker GL, D'Amore R, Allen AM, et al. Analysis of the bread wheat genome using whole-genome shotgun sequencing. Nature. 2012;491:705-10.

33. Choulet F, Alberti A, Theil S, Glover N, Barbe V, Daron J, et al. Structural and functional partitioning of bread wheat chromosome 3B. Science. 2014;345:1249721. 
34. International Rice Genome Sequencing Project. The map-based sequence of the rice genome. Nature. 2005;436:793-800.

35. Paterson AH, Bowers JE, Bruggmann R, Dubchak I, Grimwood J, Gundlach H, et al. The Sorghum bicolor genome and the diversification of grasses. Nature. 2009;457:551-6.

36. Vogel JP, Garvin DF, Mockler TC, Schmutz J, Rokhsar D, Bevan MW, et al. Genome sequencing and analysis of the model grass Brachypodium distachyon. Nature. 2010;463:763-8.

37. Ma L, Chen C, Liu X, Jiao Y, Su N, Li L, et al. A microarray analysis of the rice transcriptome and its comparison to Arabidopsis. Genome Res. 2005;15:1274-83.

38. Lu T, Lu G, Fan D, Zhu C, Li W, Zhao Q, et al. Function annotation of the rice transcriptome at single-nucleotide resolution by RNA-seq. Genome Res. 2010:20:1238-49.

39. Sekhon RS, Lin $H$, Childs KL, Hansey CN, Buell CR, de Leon $N$, et al. Genome-wide atlas of transcription during maize development. Plant J. 2011;66:553-63.

40. Sekhon RS, Briskine R, Hirsch CN, Myers CL, Springer NM, Buell CR, et al. Maize gene atlas developed by RNA sequencing and comparative evaluation of transcriptomes based on RNA sequencing and microarrays. PLoS One. 2013:8:e61005.

41. Libault M, Farmer A, Joshi T, Takahashi K, Langley RJ, Franklin LD, et al. An integrated transcriptome atlas of the crop model Glycine max, and its use in comparative analyses in plants. Plant J. 2010;63:86-99.

42. He P, Friebe B, Gill B, Zhou J-M. Allopolyploidy alters gene expression in the highly stable hexaploid wheat. Plant Mol Biol. 2003;52:401-14

43. Xu Y, Zhong L, Wu X, Fang X, Wang J. Rapid alterations of gene expression and cytosine methylation in newly synthesized Brassica napus allopolyploids. Planta. 2009;229:471-83.

44. Zou C, Lehti-Shiu MD, Thibaud-Nissen F, Prakash T, Buell CR, Shiu S-H. Evolutionary and expression signatures of pseudogenes in arabidopsis and rice. Plant Physiol. 2009;151:3-15

45. Thibaud-Nissen F, Ouyang S, Buell CR. Identification and characterization of pseudogenes in the rice gene complement. BMC Genomics. 2009; 10:317

46. Vicient C. Transcriptional activity of transposable elements in maize. BMC Genomics. 2010;11:601

47. Li W, Yang W, Wang X-J. Pseudogenes: pseudo or real functional elements? J Genet Genomics. 2013;40:171-7.

48. Wen Y-Z, Zheng L-L, Qu L-H, Ayala FJ, Lun Z-R. Pseudogenes are not pseudo any more. RNA Biol. 2012;9:27-32.

49. Liu J, Jung $C, X u$ J, Wang H, Deng S, Bernad L, et al. Genome-wide analysis uncovers regulation of long intergenic noncoding RNAs in arabidopsis. Plant Cell. 2012;24:4333-45.

50. Shuai P, Liang D, Tang S, Zhang Z, Ye C-Y, Su Y et al. Genome-wide identification and functional prediction of novel and drought-responsive lincRNAs in Populus trichocarpa. J Exp Bot. 2014; doi:10.1093/jxb/eru256.

51. Britto-Kido SA, Ferreira Neto JRC, Pandolfi V, Marcelino-Guimarães FC, Nepomuceno AL, Vilela Abdelnoor $\mathrm{R}$, et al. Natural antisense transcripts in plants: a review and identification in soybean infected with Phakopsora pachyrhizi SuperSAGE Library. Sci World J. 2013;2013:14

52. Zhang Y-C, Chen Y-Q. Long noncoding RNAs: new regulators in plant development. Biochem Biophysical Res Commun. 2013:436:111-4.

53. Wang XJ, Gaasterland T, Chua NH. Genome-wide prediction and identification of cis-natural antisense transcripts in Arabidopsis thaliana. Genome Biol. 2005;6:R30

54. Coram TE, Settles ML, Chen X. Large-scale analysis of antisense transcription in wheat using the Affymetrix GeneChip Wheat Genome Array. BMC Genomics. 2009;10:253.

55. Poole RL, Barker GLA, Werner K, Biggi GF, Coghill J, Gibbings JG, et al. Analysis of wheat sage tags reveals evidence for widespread antisense transcription. BMC Genomics. 2008:9:475.

56. Osato N, Yamada H, Satoh K, Ooka H, Yamamoto M, Suzuki K, et al. Antisense transcripts with rice full-length cDNAs. Genome Biol. 2003;5:R5.

57. Lu T, Zhu C, Lu G, Guo Y, Zhou Y, Zhang Z, et al. Strand-specific RNA-sec reveals widespread occurrence of novel cis-natural antisense transcripts in rice. BMC Genomics. 2012;13:721.

58. Zhang X, Lii Y, Wu Z, Polishko A, Zhang H, Chinnusamy V, et al. Mechanisms of small RNA generation from cis-NATs in response to environmental and developmental cues. Mol Plant. 2013;6:704-15.
59. Abranches R, Beven AF, Aragon-Alcaide L, Shaw PJ. Transcription sites are not correlated with chromosome territories in wheat nuclei. J Cell Biol. 1998;143:5-12.

60. Baker K, Bayer M, Cook N, Dreißig S, Dhillon T, Russell J, et al. The low recombining pericentromeric region of barley restricts gene diversity and evolution but not gene expression. Plant J. 2014;79:981-92.

61. Wang L, Zhao S, Gu C, Zhou Y, Zhou H, Ma J, et al. Deep RNA-Seq uncovers the peach transcriptome landscape. Plant Mol Biol. 2013;83:365-77.

62. Werner A. Biological functions of natural antisense transcripts. BMC Biol. 2013;11:31.

63. Nishizawa M, Okumura T, Ikeya Y, Kimura T. Regulation of inducible gene expression by natural antisense transcripts. Front Biosci. 2012;17:938-58.

64. Faghihi MA, Wahlestedt C. Regulatory roles of natural antisense transcripts. Nat Rev Mol Cell Biol. 2009;10:637-43.

65. Li SW, Feng L, Niu DK. Selection for the miniaturization of highly expressed genes. Biochem Biophys Res Commun. 2007;360:586-92.

66. Vinogradov AE. Compactness of human housekeeping genes: selection for economy or genomic design? Trends Genet. 2004;20:248-53.

67. Marquez Y, Brown JW, Simpson C, Barta A, Kalyna M. Transcriptome survey reveals increased complexity of the alternative splicing landscape in Arabidopsis. Genome Res. 2012;22:1184-95.

68. Walters B, Lum G, Sablok G, Min XJ. Genome-wide landscape of alternative splicing events in Brachypodium distachyon. DNA Res. 2013;20:163-71

69. Wang L, Cao C, Ma Q, Zeng Q, Wang H, Cheng Z, et al. RNA-seq analyses of multiple meristems of soybean: novel and alternative transcripts, evolutionary and functional implications. BMC Plant Biol. 2014;14:169.

70. Mayer KF, Waugh R, Brown JW, Schulman A, Langridge P, Platzer M, et al. A physical, genetic and functional sequence assembly of the barley genome. Nature. 2012:491:711-6.

71. Syed NH, Kalyna M, Marquez Y, Barta A, Brown JW. Alternative splicing in plants-coming of age. Trends Plant Sci. 2012;17:616-23.

72. Barbazuk WB, Fu Y, McGinnis KM. Genome-wide analyses of alternative splicing in plants: opportunities and challenges. Genome Res. 2008;18:1381-92.

73. Keren H, Lev-Maor G, Ast G. Alternative splicing and evolution: diversification, exon definition and function. Nat Rev Genet. 2010;11:345-55.

74. Wang BB, Brendel V. Genomewide comparative analysis of alternative splicing in plants. Proc Natl Acad Sci U S A. 2006;103:7175-80.

75. Eisenberg E, Levanon EY. Human housekeeping genes are compact. Trends Genet. 2003;19:362-5.

76. Woody JL, Shoemaker RC. Gene expression: sizing it all up. Front Genet. 2011;2:70.

77. Urrutia AO, Hurst LD. The signature of selection mediated by expression on human genes. Genome Res. 2003:13:2260-4.

78. Seoighe C, Gehring C, Hurst LD. Gametophytic selection in Arabidopsis thaliana supports the selective model of intron length reduction. PLoS Genet. 2005;1:e13.

79. Hansey CN, Vaillancourt B, Sekhon RS, de Leon N, Kaeppler SM, Buell CR. Maize (Zea mays $L$ ) genome diversity as revealed by RNA-sequencing. PLoS One. 2012;7:e33071.

80. Sémon M, Duret L. Evolutionary origin and maintenance of coexpressed gene clusters in mammals. Mol Biol Evol. 2006;23:1715-23.

81. Spellman PT, Rubin GM. Evidence for large domains of similarly expressed genes in the Drosophila genome. J Biol. 2002;1:5.

82. Chen WH, de Meaux J, Lercher MJ. Co-expression of neighbouring genes in Arabidopsis: separating chromatin effects from direct interactions. BMC Genomics. 2010;11:178.

83. Schnable JC, Springer NM, Freeling M. Differentiation of the maize subgenomes by genome dominance and both ancient and ongoing gene loss. Proc Natl Acad Sci U S A. 2011;108:4069-74.

84. Yoo MJ, Szadkowski E, Wendel JF. Homoeolog expression bias and expression level dominance in allopolyploid cotton. Heredity (Edinb) 2013;110:171-80

85. Cheng F, Wu J, Fang L, Sun S, Liu B, Lin K, et al. Biased gene fractionation and dominant gene expression among the subgenomes of Brassica rapa. PLoS One. 2012;7:e36442.

86. Parada L, McQueen P, Misteli T. Tissue-specific spatial organization of genomes. Genome Biol. 2004;5:R44.

87. Branco MR, Pombo A. Chromosome organization: new facts, new models. Trends Cell Biol. 2007:17:127-34 
88. Elcock LS, Bridger JM. Exploring the relationship between interphase gene positioning, transcriptional regulation and the nuclear matrix. Biochem Soc Trans. 2010;38:263-7

89. Dong F, Jiang J. Non-Rabl patterns of centromere and telomere distribution in the interphase nuclei of plant cells. Chromosome Res. 1998;6:551-8.

90. Cowan CR, Carlton PM, Cande WZ. The polar arrangement of telomeres in interphase and meiosis. Rabl Organization and the Bouquet. Plant Physiol. 2001;125:532-8.

91. Santos AP, Abranches R, Stoger E, Beven A, Viegas W, Shaw PJ. The architecture of interphase chromosomes and gene positioning are altered by changes in DNA methylation and histone acetylation. J Cell Sci. 2002;115:4597-605.

92. FastQC. [http://www.bioinformatics.babraham.ac.uk/projects/fastqc]

93. Trapnell C, Williams BA, Pertea G, Mortazavi A, Kwan G, van Baren MJ, et al. Transcript assembly and quantification by RNA-Seq reveals unannotated transcripts and isoform switching during cell differentiation. Nat Biotechnol. 2010;28:511-5.

94. TopHat. [http://ccb.jhu.edu/software/tophat/index.shtml]

95. Langmead B, Trapnell C, Pop M, Salzberg SL. Ultrafast and memory-efficient alignment of short DNA sequences to the human genome. Genome Biol. 2009;10:R25.

96. Li H, Handsaker B, Wysoker A, Fennell T, Ruan J, Homer N, et al. The sequence alignment/Map format and SAMtools. Bioinformatics. 2009;25:2078-9.

97. Cufflinks. [http://cole-trapnell-lab.github.io/cufflinks/]

98. Yu J, Hu S, Wang J, Wong GK, Li S, Liu B, et al. A draft sequence of the rice genome (Oryza sativa L. ssp. indica). Science. 2002;296:79-92.

99. Haas BJ, Papanicolaou A, Yassour M, Grabherr M, Blood PD, Bowden J, et al. De novo transcript sequence reconstruction from RNA-seq using the Trinity platform for reference generation and analysis. Nat Protoc. 2013;8:1494-512.

100. Changepoint-package. [http://www.inside-r.org/packages/cran/changepoint/ docs/changepoint]

101. R software. [www.r-project.org]

102. Hierarchical clustering explorer software. [http://www.cs.umd.edu/hcil/hce/]

103. Lin W, Chen Y, Ho J, Hsiao C. GOBU: toward an integration interface for biological object. J Information Sci Eng. 2006;22:19-30.

\section{Submit your next manuscript to BioMed Central and take full advantage of:}

- Convenient online submission

- Thorough peer review

- No space constraints or color figure charges

- Immediate publication on acceptance

- Inclusion in PubMed, CAS, Scopus and Google Scholar

- Research which is freely available for redistribution 